\begin{tabular}{|c|c|}
\hline Citation/Reference & $\begin{array}{l}\text { Hunyadi B., Tousseyn S., Dupont P., Van Huffel S., De Vos M., Van } \\
\text { Paesschen W., “' } \text { A prospective fMRI-based technique for localising the } \\
\text { epileptogenic zone in presurgical evaluation of epilepsy", Neuroimage, vol. } \\
\text { 113, Jun. 2015, pp. } 329-339\end{array}$ \\
\hline Archived version & $\begin{array}{l}\text { Author manuscript: the content is identical to the content of the } \\
\text { published paper, but without the final typesetting by the publisher }\end{array}$ \\
\hline Published version & $\begin{array}{l}\text { insert link to the published version of your paper } \\
\text { http://dx.doi.org/10.1016/i.neuroimage.2015.03.011 }\end{array}$ \\
\hline Journal homepage & http://www.journals.elsevier.com/neuroimage/ \\
\hline Author contact & $\begin{array}{l}\text { Borbala.hunyadi@esat.kuleuven.be } \\
+32(0) 16321799\end{array}$ \\
\hline IR & https://lirias.kuleuven.be/handle/123456789/488740 \\
\hline
\end{tabular}




\title{
A prospective fMRI-based technique for localising the epileptogenic zone in presurgical evaluation of epilepsy
}

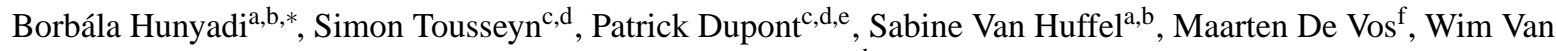 \\ Paesschen ${ }^{\mathrm{c}, \mathrm{d}}$ \\ ${ }^{a}$ STADIUS Center for Dynamical Systems, Signal Processing and Data Analytics, Department of Electrical Engineering (ESAT), KU Leuven, \\ Kasteelpark Arenberg 10, 3001, Leuven, Belgium \\ biMinds Medical IT, Leuven, Belgium \\ ${ }^{c}$ Laboratory for Epilepsy Research, UZ Leuven and KU Leuven, Herestraat 49, 3000, Leuven, Belgium \\ ${ }^{d}$ Medical Imaging Research Center, UZ Leuven and KU Leuven, Herestraat 49, 3000, Leuven, Belgium \\ ${ }^{e}$ Laboratory for Cognitive Neurology, UZ Leuven and KU Leuven, Herestraat 49, 3000, Leuven, Belgium \\ ${ }^{f}$ Institute of Biomedical Engineering, Department of Engineering Science, University of Oxford, Old Road Campus Research Building, \\ Headington, Oxford $O X 37 D Q, U K$
}

\begin{abstract}
There is growing evidence for the benefits of simultaneous EEG-fMRI as a non-invasive localising tool in the presurgical evaluation of epilepsy. However, many EEG-fMRI studies fail due to the absence of interictal epileptic discharges (IEDs) on EEG. Here we present an algorithm which makes use of fMRI as sole modality to localise the epileptogenic zone (EZ). Recent studies using various model-based or data-driven fMRI analysis techniques showed that it is feasible to find activation maps which are helpful in the detection of the EZ. However, there is lack of evidence that these techniques can be used prospectively, due to (a) their low specificity, (b) selecting multiple activation maps, or (c) a widespread epileptic network indicated by the selected maps. In the current study we present a method based on independent component analysis and a cascade of classifiers that exclusively detects a single map related to interictal epileptic brain activity. In order to establish the sensitivity and specificity of the proposed method, it was evaluated on a group of 18 EEG-negative patients with a single well-defined EZ and 13 healthy controls. The results show that our method provides maps which correctly indicate the EZ in several $(N=4)$ EEG-negative cases but at the same time maintaining a high specificity $(92 \%)$. We conclude that our fMRI-based approach can be used in a prospective manner, and can extend the applicability of fMRI to EEG-negative cases.
\end{abstract}

Keywords: presurgical evaluation, fMRI, ICA, LS-SVM

\section{Introduction}

Epilepsy, the second most common neurological disorder after stroke, occurs in over $0.5 \%$ of the world population (De Boer et al. (2008)). It is a chronic neurological disorder characterised by recurrent epileptic seizures (Blume et al. (2001)). A seizure is defined as the transient occurrence of signs and/or symptoms due to abnormal excessive or synchronous neuronal activity in the brain (Fisher et al. (2005)).

Approximately $30 \%$ of epilepsy patients are nonresponsive to anti-epileptic drugs (Engel (1996)), a condition called refractory epilepsy, and continue to suffer

*Corresponding author e-mail: bhunyadi@esat.kuleuven.be, tel.: +3216321799 fax: +32 $\overline{16321970}$ from seizures. As their quality of life is seriously compromised, surgical resection can be considered.

The final goal of the presurgical evaluation is to delineate the epileptogenic zone. The definition states that the total resection or disconnection of the epileptogenic zone is necessary and sufficient for seizure freedom (Rosenow and Lüders (2001)). However, the epileptogenic zone is a hypothetical region, i.e. there is no diagnostic modality which can directly delineate it. One has to infer to its location indirectly by defining several other relevant zones, which are involved in generating the epileptic disorder or its electrographical and clinical symptoms. The area in the cortex generating interictal epileptiform discharges is called the irritative zone. The portion of the irritative zone which produce repetitive spikes strong enough to cause clinical symptoms is the 
seizure onset zone. It often provides an accurate definition of the EZ, however, the latter may be less or more extensive in some cases. In this study we use the irritative zone as a surrogate for the EZ, hence, we will use these terms interchangeably.

Since the 1950s ictal EEG recordings are routinely used and still remain the gold standard for defining the seizure onset zone (Panayiotopoulos (2005); Rosenow and Lüders (2001)) and it has a localizing power in about $70 \%$ of cases (Foldvary et al. (2001)). However, as seizures occur infrequently compared with interictal epileptic discharges, presurgical evaluation relying on ictal recordings is a time consuming procedure. Seeking an alternative, many researchers have been investigating the utility of EEG-correlated fMRI analysis (Gotman et al. (2006); Salek-Haddadi et al. (2006)). Simultaneously recorded EEG-fMRI during interictal periods potentially provides high spatial and temporal resolution information on the irritative zone. Its clinical utility within presurgical evaluation has been demonstrated in several studies. It can improve source localisation in complex cases, such as an unclear focus on EEG or presumed multifocality (Zijlmans et al. (2007)). Moreover, some studies suggest that certain EEG-fMRI activation patterns in focal epilepsy may be an indicator of surgical outcome (An et al. (2013); Thornton et al. (2011)). Both a high sensitivity and high specificity is crucial for the clinical implementation of this technique. In a recent study we have formulated a guideline for different analysis and interpretation settings for spike-related EEG-fMRI (Tousseyn et al. (2014a)). It was shown that the unique cluster containing the maximal significant BOLD activation is a sensitive (57\%) and specific (100\%) marker of the EZ.

Despite the promising advances in the field, 40-70\% of EEG-fMRI studies fail today, due to the absence of interictal discharges in the EEG, or the lack of significant BOLD signal changes correlated to their timing (Grouiller et al. (2011)). A promising approach to resolve this issue is the automatic marking of EEG events presumably related to the activity of the epileptic source based on patient-specific topographic maps (Grouiller et al. (2011)). Although hemodynamic correlates of such scalp maps were reported in the majority of previously inconclusive cases, the proposed technique is not specific for epilepsy related activity (Tousseyn et al. (2014b)). The specificity, and, therefore, the clinical applicability can be improved by taking into account the morphological characteristics of the patient-specific spike template as well (Tousseyn et al. (2014b)). Nevertheless, epileptic activity of deep structures remain undetected in EEG, moreover, severe artefacts related to the magnetic field of the scanner hinder the interpretation of the recordings. Although several techniques exist to reduce these artefacts, still, the manual marking of epileptic events requires a high level of vigilance (Nonclerca et al. (2012)), it is a time-consuming and subjective procedure (Zijlmans et al. (2007)). Therefore, techniques which can infer to the localization of the epileptic sources solely based on fMRI without the need of EEG information are highly desirable.

The first such algorithm, temporal clustering analysis (TCA) (Morgan et al. (2004)) aimed to detect irregular, transient fMRI activation signals. Unfortunately, this method was not only sensitive to interictal epileptic discharges, but to motion artefacts and physiological noise (Hamandi et al. (2005)). Therefore, an improved technique, called 2dTCA was developed (Morgan et al. (2008)), which is capable of detecting multiple timing patterns of transient BOLD activations. However, only limited evidence was presented from real epileptic fMRI signals to validate the method. More extensive validation and better performance was reported by (Lopes et al. (2012)) using an algorithm based on the activelet representation of the fMRI time course. Activelets are a recently developed wavelet basis (Khalidov et al. (2011)), constructed based on the linear approximation of the balloon model (Buxton et al. (1998)) of the hemodynamic response function. As such, the BOLD signal in response to a transient neural activation is sparsely represented in this basis. After estimating the timing of transient neural activation, voxels showing similar activity were gathered using spatiotemporal clustering. The cluster with the sparsest temporal pattern successfully identified epileptic BOLD activations (Lopes et al. (2012)).

The above model-based techniques rely on the assumption that spikes have sparse, transient behaviour. However, some patients show up to 2000 spikes per hour (Tousseyn et al. (2014a)), i.e. these model assumptions do not always hold. Data-driven approaches such as independent component analysis (ICA) are more flexible and potentially perform well under various circumstances. Moreover, ICA-based techniques have the advantage of adaptively handling artefacts, capturing them in individual components.

Independent component analysis has been applied to ictal (Hunvadi et al. (2013); Leite et al. (2013); LeVan et al. (2010); Thornton et al. (2010)) and interictal (Leite et al. (2013); Moeller et al. (2011); Rodionov et al. (2007)) fMRI time series. Although independent components (ICs) can be automatically sorted (Rodionov et al. (2007); Thornton et al. (2010)) into BOLD-related or various artefact-related groups 
using supervised classification (De Martino et al. (2007)), the epileptic IC still has to be identified in a retrospective manner, or based on the simultaneously recorded EEG.

In a previous study (Hunyadi et al. (2014)) we presented a novel approach to fully automate this procedure to select the epileptic IC from EEG-positive cases, i.e in cases where interictal spikes were visible on the simultaneously recorded EEG. However, the proposed method is especially beneficial in patients where no interictal discharges are visible in the EEG. In Hunyadi et al. (2013) we have shown that epileptic ICs can be found in such EEG-negative cases as well. Therefore, the goal of the current study was to validate the proposed method in a group of EEG-negative patients. Aiming for a prospective technique which is applicable in clinical practice, we put an emphasis on developing a highly specific method. Therefore, our technique is designed to be exclusive, rather than inclusive in contrast with a recent approach based on ICA (Zhang et al. (2014)).

The paper is organised as follows. In section 2.1 the patient dataset and the data collection is described. In 2.2 we give an overview about the proposed methodology. Detailed explanation, i.e. the features extracted to characterise the fMRI ICs and the supervised classification approach used to select the epileptic ICs, follows in sections 2.3 and 2.4. Subsequently, section 2.5 discusses the interpretation of the resulting maps and the criteria to evaluate our results. Section 3 presents the outcome of the automatic selection method in EEGnegative patients and in a group of healthy controls. Finally, section 4 and 5 are devoted to discussion and conclusions.

\section{Materials and Methods}

\subsection{Data collection}

For the purpose of this study patient data were included based on the following criteria: (1.1) consecutive adults who underwent a full presurgical evaluation for refractory focal epilepsy between August 2010 and November 2013, (1.2) concordant data pointing to one epileptic focus using all presurgical investigations except EEG-fMRI, and (1.3) good surgical outcome (ILAE 1-3) 1 with at least 6 month follow-up. For including patients in the training set for the ICA selection

\footnotetext{
${ }^{1} 1$, completely seizure-free; 2 , only auras; 3 , one to three seizure days per year auras; 4 , four seizure days per year to $50 \%$ reduction of baseline seizure days auras; $5,<50 \%$ reduction of baseline seizure days to $100 \%$ increase of baseline seizure days auras; 6 , more than $100 \%$ increase of baseline seizure days auras)
}

algorithm, additional criteria were applied, namely (2.1) interictal epileptic discharges were present on the EEG, (2.2) the outcome EEG-informed fMRI was in agreement with the result of the presurgical work-up based on all other imaging and diagnostic data, and (2.3) no brain-deforming lesions were present. The algorithm was tested on a dataset including patients based on the following criteria additional to 1.1-1.3: (3.1) no interictal epileptic discharges were present on the simultaneously recorded EEG.

The final training set and test set consisted of the interictal fMRI time series of 12 and 18 patients, respectively.

In addition, fMRI data from 13 healthy individuals were recorded and included in the study as well, in order to assess the behaviour of the proposed method in the absence of epileptic activity.

For practical reasons, the number and length of the fMRI runs (recording sessions) varied among patients. In case there were multiple or long runs, only the first 270 consecutive images of the first run of the training patients and healthy control subjects were included in the dataset. This is particularly important for the training phase, in order to avoid that the algorithm gets biased towards characteristics of patients where larger amount of data was available. For test subjects all available runs comprising 270 images were included.

Functional images were acquired using a whole-brain single-shot T2* Gradient-Echo Echo Planar Imaging sequence in one of two 3 Tesla MR-scanners (Achieva TX with a 32-channel head coil and Intera Achieva with an 8-channel head coil, Philips Medical Systems, Best, The Netherlands); echo time $=33 \mathrm{~ms}$, repetition time 2.2-2.5 s, voxel size: $2.6 \times 3 \times 2.6 \mathrm{~mm}^{3}$.

All fMRI images were realigned, slice-time corrected, normalised to MNI space using the coregistered high-resolution structural scan (resampled voxel size $2 \times 2 \times 2 \mathrm{~mm}^{3}$ ) and spatially smoothed with an isotropic Gaussian kernel of $6 \mathrm{~mm}$ full width at half maximum using statistical parametric mapping (SPM8, Wellcome Department of Imaging Neuroscience, University College London, UK; available at http://www.fil.ion.ucl.ac.uk/spm/). Subsequently, EEG-correlated fMRI analysis was performed in the EEG-positive cases within the general linear model (GLM) framework. The timing of the preponderant IEDs convolved with the canonical hemodynamic response function (provided in SPM8) was used as a regressor of interest. The six rigid-body motion correction parameters, the fMRI signal averaged over the lateral ventricles, and the signal averaged over a region within the white matter were included as 
confounding covariates. The activation maps were thresholded at a significance level of $p<0.05$ with family wise error correction.

\subsection{Blind selection method: overview}

The methodology used in this study and preliminary results on EEG-positive cases have been presented in (Hunyadi et al. (2014)). Below we explain in more detail the various steps and most important considerations of the methodology. A flowchart depicting the various steps involved in the proposed algorithm is shown in figure 1

As a first step, ICA was performed on the fMRI time series. Consecutively, a cascade of two classifiers was applied. The first discrimination stage discarded the ICs related to artefacts. This stage is denoted by Feature extraction 1 and Classification 1 steps in the flowchart. Afterwards, using the second classifier, involving Feature extraction 2 and Classification 2 steps, the epileptic IC was selected from the remaining reduced set of ICs consisting of BOLD signal related ICs. Finally, localisation information was retrieved from the spatial map corresponding to the selected epileptic IC.

The Fix plug-in of the FSL toolbox (http://fsl.fmrib.ox.ac.uk/fsl/fslwiki/FIX) was used to perform the ICA, the Feature extraction 1 and the Classification 1 steps, which aim to discriminate between BOLD and artefact related ICs. The number of independent components was automatically estimated within the toolbox using Bayesian dimensionality estimation. Detailed explanation about the extracted features and the applied machine learning technique is given in (Salimi-Khorshidi et al. (2014)). Our contribution is the development of the features and the classifier for the second discrimination step (Feature extraction 2 and Classification 2 steps) in the cascade. These steps are elaborated upon in the following sections.

\subsection{Feature extraction}

In this section we describe and motivate the measures computed within the Feature extraction 2 step.

The goal of this study was to automatically select epileptic independent components which can be of use in presurgical evaluation in a prospective manner. In (Hunyadi et al. (2013)) we argued that there might exist multiple epileptic sources, which correspond to partially overlapping parts of the epileptic network and reflect different aspects of epileptic activity. However, the clinically most relevant finding would be the automatic recognition of a single epileptic IC. Furthermore,

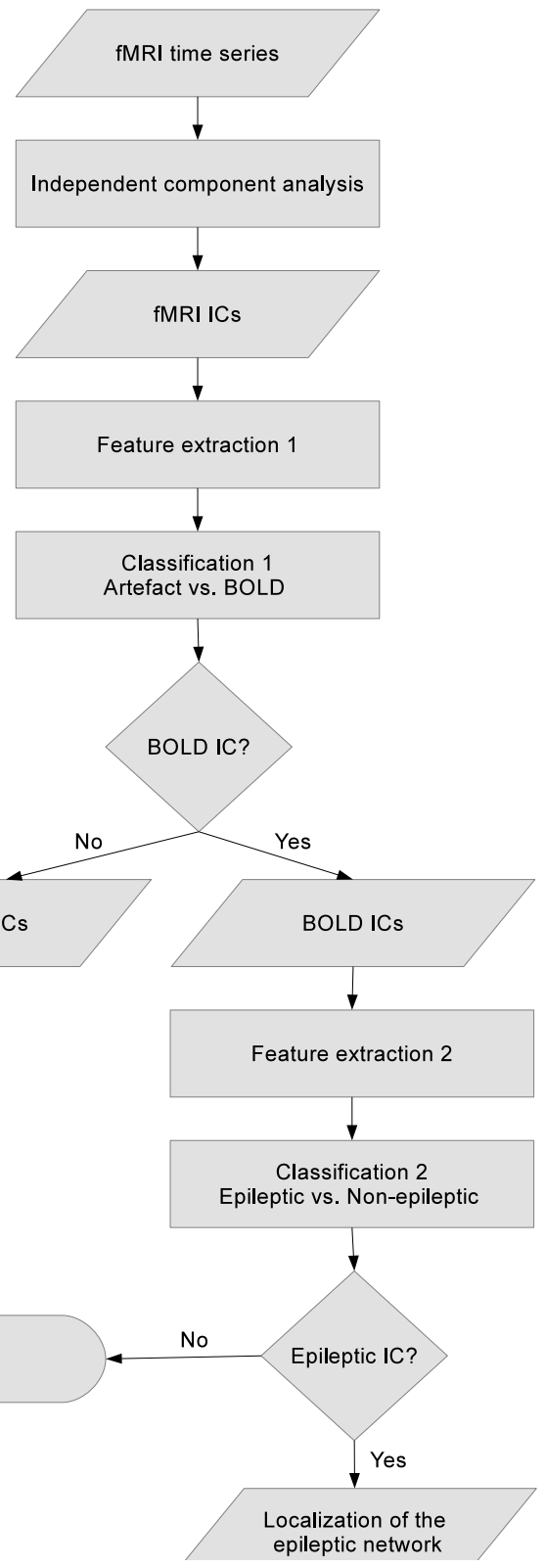

Figure 1: The proposed algorithm for automatic localisation of the EZ involves several steps. First, ICA is performed on the fMRI time series. Consecutively, a cascade of classifiers is applied. First, in the Feature extraction 1 and Classification 1 steps, artefact related ICs are discarded as described in (Salimi-Khorshidi et al. (2014)). Afterwards, in the Feature extraction 2 and Classification 2 steps, the epileptic IC is selected from the remaining reduced set of BOLD related ICs (Hunyadi et al. (2014)). Finally, localisation information is retrieved from the spatial map corresponding to the epileptic IC. 
for the prospective use of the proposed technique it is important that the selected IC unambiguously points to a single brain region, which, consequently, can be attributed to the EZ. Therefore, we concentrate on characteristics which are particular to such ideal components. Moreover, we reason that the fMRI ICs remaining after the first classification step are expected to correspond to either epilepsy related or to resting state network (RSN) related sources. Accordingly, the extracted features should reflect characteristics which are systematically different among ICs belonging to these groups. Based on these considerations, the following features were extracted from the fMRI ICs. For the exact definition of these features, see Appendix.

Number of clusters. Suprathreshold voxels in the spatial map corresponding to an IC are spatially organised in one or more clusters. The number of clusters in an epileptic IC is ideally 1, corresponding to the EZ. In contrast, various RSNs consist of multiple active regions.

Asymmetry. The EZ of a unifocal epilepsy patient is usually restricted to a region in strictly one hemisphere, thus, will show asymmetry. In contrast, RSN components in general involve bilateral regions and, as such, are more symmetric.

Sparsity in activelet basis. Activelets are a dictionary of wavelet basis functions which were developed specifically in order to fit the characteristics of the BOLD signal in response to a sparse, transient neural event (Khalidov et al. (2011). As such, signals comprising sparse transient events, such as interictal epileptic spikes, will have sparse representation in the activelet basis.

Sparsity in sine dictionary. The time course of resting state networks is characterised by low-frequency (0.01$0.1 \mathrm{~Hz}$ ) fluctuations (Cordes et al. (2001)). Therefore, they are expected to have a sparse representation in a sine dictionary restricted to this frequency band.

We have investigated the discriminative power of these features in (Hunyadi et al. (2014)). We showed that these features show significant $(p<0.05$, asymmetry, sparsity in activelet and sine basis) or marginally insignificant ( $p<0.1$, number of clusters) differences across the epileptic and non-epileptic BOLD ICs.

\subsection{Classification}

In this section we describe and motivate the machine learning approach used in the Classification 2 step.
Based on the above extracted features, a leastsquares support vector machine (LS-SVM) classifier was trained to differentiate between epileptic and nonepileptic ICs. The class of epileptic ICs consisted of the ICs of EEG-positive patients showing the largest overlap with the cluster containing the maximally activated voxel in the GLM-based fMRI activation map in each patient. It has been shown that the cluster containing the maximal significant activation identifies best the EZ in a widespread interictal network obtained using GLM (Tousseyn et al. (2014a)). In addition, ICs which showed at least $10 \%$ overlap with the same cluster and significantly correlated in time with the occurrence of the IEDs, were also included in the epileptic class. All other ICs of EEG-positive patients were included in the class of non-epileptic ICs.

Note that our final goal is to select an IC which indicates the EZ, nevertheless, we chose to define the training examples based on the GLM-based activation maps and not based on the surgical resection zone for the following reason. In this study interictal processes were recorded in the fMRI. We do expect that the fMRI-based maps will contain the EZ, but perfect overlap is unlikely. The GLM-based fMRI maps provide a more reliable image of how the epileptic ICs should look like.

Recall that LS-SVM takes decisions according to:

$$
y(\mathbf{x})=\operatorname{sign}\left(\mathbf{w}^{T} \varphi(\mathbf{x})+b\right),
$$

with $y(\mathbf{x}) \in\{-1,1\}$ are the class labels (non-epileptic and epileptic, respectively), $\mathbf{x}$ contains the extracted features for each IC, $\mathbf{w}$ is a weighting vector, $b$ is a bias term and $\varphi$ is a feature mapping. Note that this formula might assign multiple ICs to the epileptic class, however, we were interested in selecting exactly one epileptic IC in each patient. Therefore, we modified the decision function as follows:

$y\left(\mathbf{x}_{i}\right)= \begin{cases}1 & \text { if } \arg \max _{\mathbf{x}}\left(\mathbf{w}^{T} \varphi(\mathbf{x})\right)=i \text { and } \mathbf{w}^{T} \varphi(\mathbf{x})+b>0 \\ 0 & \text { otherwise. }\end{cases}$

The epileptic ICs were classified for each patient individually, therefore, at most one IC was selected in each patient. The values $\mathbf{w}^{T} \varphi(\mathbf{x})$ determine a ranking of the ICs, the highest value corresponding to the IC which resembles most the epileptic ICs in the training data. In case this value exceeded the threshold $-b$, the first ranked IC was selected as epileptic. Otherwise, if no IC showed enough resemblance to the training epileptic ICs, no selection was made.

There are a few parameters to choose prior to classifier training. First, an optimal threshold value has to 
be determined for the first classification step (artefact vs. BOLD using the FIX toolbox). As the outcome of this classification is a probability that each IC belongs to one class or another, this threshold represents a trade-off between rejecting bad components and keeping good ones. A higher threshold will reject more components, therefore, the second classification step will have to differentiate between fewer components. Furthermore, a feature mapping or kernel function has to be chosen for LS-SVM. We have considered both a linear and an RBF-kernel. These parameters were determined in a leave-one-patient-out crossvalidation setting using the training data, optimised for accuracy. The optimal choice turned out to be a conservative threshold of 40 (Salimi-Khorshidi et al. (2014)) for the FIX classification step, and a linear kernel for the second classifier.

Using these parameter choices, the classifier was retrained using all EEG-positive cases and then applied on all EEG-negative cases.

\subsection{Evaluation criteria}

Interpretation of the resulting maps. The selected IC maps were interpreted as follows. First, the voxel values in each spatial map were converted to z-scores, by subtracting the mean of all voxel values and dividing these by their standard deviation. Then, the z-scored maps were thresholded at 5, a value as established in (Hunyadi et al. (2013)) using a partially independent patient group (5 and 7 overlapping training and test patients, respectively). Finally, only clusters containing at least 100 voxels were retained. This threshold was set in order to match the value used in the definition of our feature " "number of clusters"" (see Appendix). The spatial maps obtained in this way were categorised as follows:

1. Informative: In order to call an IC map informative, the following criteria had to be met. The IC had to contain a single cluster inside the brain. Moreover, if ICs were selected from multiple runs of the same patient, these had to point to the same anatomical region. In other words, all selected ICs of all sessions had to be concordant in order to come to a conclusion. Agreement of the selected ICs across sessions was determined visually, based on whether the suprathreshold clusters point to the same anatomical region (e.g. left mesial temporal lobe / insula / right occipital lobe, etc.) In case there was a selection in one session and no selection in another session, this was considered informative: no selection only means that there was no detectable epileptic activity during that specific session either due to the lack of activity, or due to low sensitivity. Nevertheless, such maps unambiguously suggest a certain epileptogenic zone, which could be used in the presurgical evaluation. Within the informative category, the maps were assigned to either of the groups below:

(a) Correct: The epileptogenic zone suggested based on the selected ICs was concordant with the actual resection zone.

(b) Misleading: The epileptogenic zone suggested based on the selected ICs was not concordant with the actual resection zone.

2. Non-informative: In case a map did not meet the criteria to be informative, it was assigned to the non-informative category. More specifically, noninformative ICs were the ones which contained multiple clusters or a cluster outside the brain. Furthermore, if ICs were selected from multiple runs of the same patient and these pointed to different anatomical regions, the results are also noninformative. Such maps are ambiguous, therefore, can not be interpreted within the presurgical evaluation independently from other modalities.

Note that the categories informative and noninformative were determined prospectively. However, correct and misleading categories were determined retrospectively, and serve the purpose of evaluating our results.

Evaluation measures. Based on the above interpretation of the maps, the following measures for binary classification were established:

- true positive (TP): number of patients where a correct IC was selected

- false negative (FN): number of patients where a misleading, a non-informative or no selection was made

- true negative (TN): number of healthy controls where no selection was made

- false positive (FP): number of healthy controls where a selection was made

Subsequently, the evaluation measures for binary classification were computed:

- accuracy: $A C C=\frac{T P+T N}{T P+T N+F P+F N}$

- sensitivity: $S E N S=\frac{T P}{T P+F N}$

- specificity: $S P E C=\frac{T N}{T N+F P}$ 


\section{Results}

ICA extracted an average of $57.7 \pm 7.3$ components over all runs of all test patients. The first classification step rejected $63 \%$ of components, therefore, on average $21.2 \pm 6.1$ BOLD signal related components remained.

During the second classification step a selection was made in 11 out of 18 test patients. The selected IC was informative, i.e. consisted of 1 suprathreshold cluster inside the brain, in 4 cases. These maps are shown in Figure 2 in yellow. In case of patient 1 , where a selection was made in 2 runs, the second selected IC is shown in green. Notably, these 2 maps show very high similarity. In all 4 cases the selected IC was correct, i.e. it was concordant with the resection zone. The resection zone of patient 11 , delineated based on the postoperative MRI, is shown in red. The overlap between the selected map and the resection zone is shown in orange. No post-operative MRI was available for patients 1 , and 12. In these cases surgical resection in the left and right temporal lobe was reported, respectively.

No misleading maps were selected.

In 7 cases the selected ICs were not informative. In patient 4 the selected IC consisted of a single suprathreshold cluster outside the brain, as shown on Figure 3c In 6 further cases 2 , in patients 6, 7, $8,10,14$ and 17, the selected IC maps consisted of several clusters. Interestingly, these maps were very similar to each other and showed activity in areas known to be part of the executive control network (ECN) (Shirer et al. (2012)). Interestingly, the individual maps were highly lateralised ipsilateral to the resection zone, except for patient 17. Group average maps, obtained by taking the mean of the voxel values over all the left and right lateralised maps, are shown in Figure $3 \mathrm{a}$ and $3 \mathrm{~b}$, respectively. For comparison, atlases of the left and right ECN, available at http://findlab.stanford.edu/research, are also visualised.

A summary of the patient-by-patient outcomes for the test patients are shown in Table 1

A selection was made in 1 out of 13 control subjects. The selected map is shown in Figure 3d The map was selected probably due to the fact that it is highly lateralised.

Quantitatively speaking, the proposed method reaches an accuracy of $51,6 \%$, a sensitivity of $22 \%$ and a specificity of $92 \%$ on the test group of EEG-negative patients.

\footnotetext{
${ }^{2} \mathrm{~A}$ selection was made in 2 runs in patients 6,8 and 10 .
}

\section{Discussion}

We proposed a novel approach for determining the epileptogenic zone in the presurgical evaluation of epilepsy, based on fMRI as a single modality.

The proposed method delivered 4 informative maps in 18 patients (22\% sensitivity). All 4 maps correctly pointed to the epileptogenic zone, i.e. a successful surgical resection had been performed in the same region based on a complementary presurgical evaluation, rendering the patients seizure free. Although an indication was formulated in only a relatively small percentage of cases, our method has significant advantages. It is completely automatic as only a single IC map is selected, and only unambiguous maps indicating a single region of epileptic activity are taken into consideration.

We compared our results with the reported performance of two recently developed algorithms, the activelet-based spatiotemporal clustering method (Lopes et al. (2012)) and another ICA-based approach (Zhang et al. (2014)). Although the results are not directly comparable as they were obtained on different datasets, some essential aspects are worth to consider. The ICA-based method (Lopes et al. (2012)) performs very well in EEG-positive cases. However, only limited validation was available for EEG-negative runs. In 5 runs without events on the EEG recorded in 2 patients, 3 maps provided concordant results with the patients' diagnostic, while 2 maps did not. The question arises how to handle contradictory activation maps from different runs of the same patient. The method (Zhang et al. (2014)) was validated on 10 patients where only fMRI data was recorded. A selection was made in 9 patients, 7 of which were concordant with the patients' diagnostics. However, besides the concordant epileptic map some discordant maps were also selected in 3 , rendering these cases non-informative. Therefore, (Zhang et al. (2014)) reaches an accuracy of $40 \%$ compared to $52 \%$ in our method. Furthermore, the proposed method selected potentially epilepsy related maps in 3 out of 7 healthy controls in resting state. This corresponds to a specificity of $57 \%$ compared to $92 \%$ in our approach. Moreover, in both (Lopes et al. (2012)) and ((9 Zhang et al. (2014)) the selected epileptic maps contained multiple activation clusters. This means that the epileptogenic zone could not be objectively defined based on the selected maps. Although the sensitivity of our method was relatively low, our approach was more specific and fully objective, therefore, it can potentially be used prospectively as opposed to the methods proposed in the above papers. Moreover, we would like to emphasise that the goal of the proposed approach is not to replace tradi- 


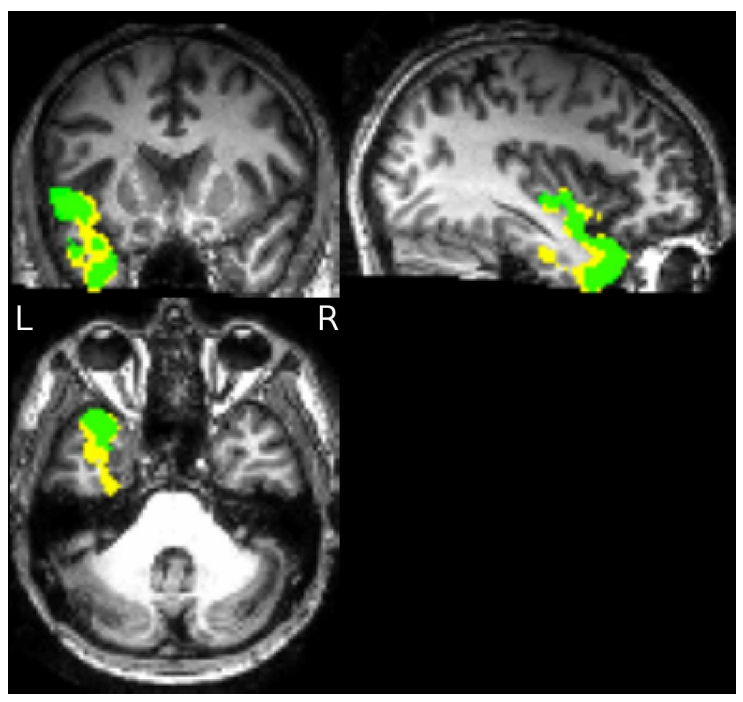

(a) Patient 1

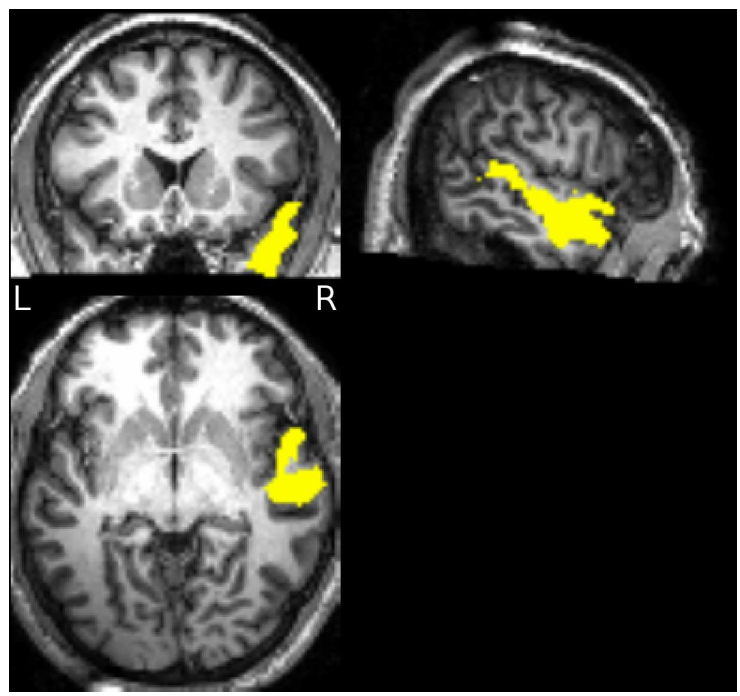

(c) Patient 12

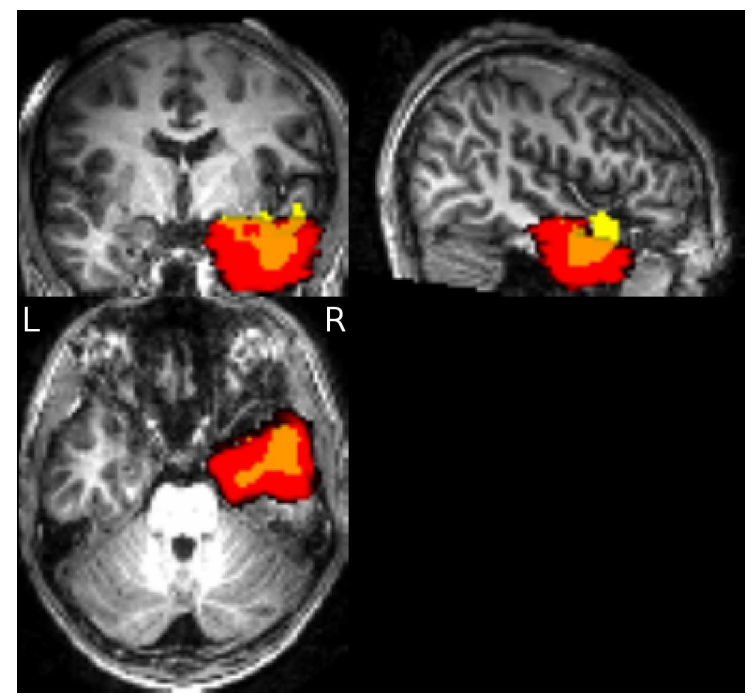

(b) Patient 11

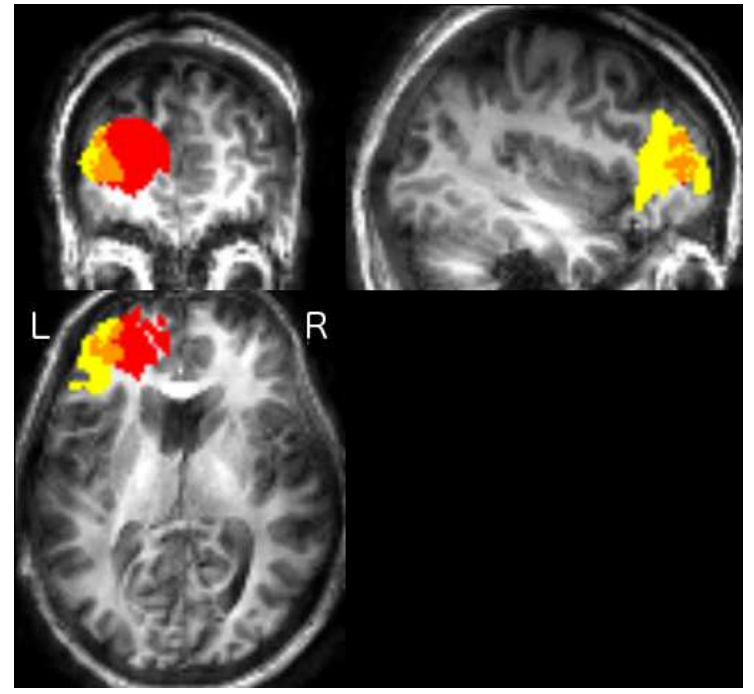

(d) Patient 13

Figure 2: Informative IC maps selected in patients. In 4 cases the selected IC was informative and concordant with the resection zone. The selected IC maps are shown in yellow. In case of patient 1 , where a selection was made in 2 runs, the second selected IC is shown in green. The resection zone of patient 11, delineated based on the postoperative MRI, is shown in red. The overlap between the IC map and the resection zone is shown in orange. No postoperative MRI was available for patients 1 and 12. In these cases surgical resection in the left temporal lobe and right temporal lobe was reported, respectively. 
Table 1: Detailed information about the diagnostics, imaging results, fMRI recordings and the outcome of the proposed method for the EEG-negative patients. The following abbreviations are used in the table. n.a.: not available; HS: hypocampal sclerosis; FCD: foal cortical dysplasia; DNET: dysembryoplastic neuroepithilial tumor

\begin{tabular}{|c|c|c|c|c|c|c|c|c|c|c|}
\hline Patient & Resection Zone & Structural lesion & SISCOM & FDG-PET & Pathology & \# fMRI runs & $\begin{array}{l}\text { average\# } \\
\text { ICs/run }\end{array}$ & $\begin{array}{l}\text { average\# } \\
\text { BOLD ICs/run }\end{array}$ & Selected IC & $\begin{array}{l}\text { Lateralisation } \\
\text { of selected IC }\end{array}$ \\
\hline 1 & $\begin{array}{ll}\begin{array}{l}\text { Left } \\
\text { lobe }\end{array} & \text { temporal } \\
\end{array}$ & $\begin{array}{ll}\text { Left } & \text { temporal } \\
\text { DNET } & \\
\end{array}$ & n.a. & left temporal lobe & DNET & $\overline{44}$ & "59.75 & 24.00 & Correct & 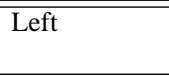 \\
\hline 2 & Right insula & right insula & $\begin{array}{l}\text { temporooccipital, } \\
\text { right frontal }\end{array}$ & $\begin{array}{l}\text { parietal }(\mathrm{R}>\mathrm{L}) \text {, } \\
\text { temporal }(\mathrm{R}>\mathrm{L}) \text {, } \\
\text { occipital }\end{array}$ & cortical displasia & 4 & 67.00 & 28.50 & No selection & \\
\hline 3 & $\begin{array}{ll}\text { Left } & \text { temporal } \\
\text { lobe }\end{array}$ & left HS & $\begin{array}{l}\text { Bitemporal } \\
(\mathrm{R}>\mathrm{L})\end{array}$ & n.a. & left temporal HS & 2 & 57.00 & 25.50 & No selection & \\
\hline 4 & $\begin{array}{l}\text { Right temporal } \\
\text { lobe }\end{array}$ & $\begin{array}{l}\text { right temporal } \\
\text { cavernoma }\end{array}$ & right temporal & bilateral temporal & angioma & 6 & 65.67 & 34.17 & Not informative $_{\text {- }}^{a}$ & Right \\
\hline 5 & $\begin{array}{l}\text { Left } \\
\text { lobe }\end{array}$ & Normal & left temporal & left temporal & FCD & 2 & 43.00 & 22.00 & No selection & \\
\hline 6 & $\begin{array}{l}\text { Right temporal } \\
\text { lobe }\end{array}$ & right $\mathrm{HS}$ & right temporal & right temporal & $\mathrm{HS}$ & 4 & 70.25 & 21.75 & Not informative ${ }^{b}$ & Right \\
\hline 7 & $\begin{array}{l}\text { Right temporal } \\
\text { lobe }\end{array}$ & right $\mathrm{HS}$ & $\mathrm{n} / \mathrm{a}$ & right temporal & $\mathrm{HS}$ & 6 & 55.83 & 23.30 & Not informative $_{\text {- }}^{c}$ & Right \\
\hline 8 & $\begin{array}{ll}\begin{array}{l}\text { Left } \\
\text { lobe }\end{array} & \text { temporal } \\
\end{array}$ & left HS & left temporal & n.a. & left temporal HS & 6 & 49.50 & 11.30 & Not informative & Left \\
\hline 9 & $\begin{array}{l}\text { Left temporal } \\
\text { neocortex }\end{array}$ & $\begin{array}{l}\text { left temporal neo- } \\
\text { cortical }\end{array}$ & n.a. & $\begin{array}{l}\text { left temporal hy- } \\
\text { permetabolism, } \\
\text { hypometabolism } \\
\text { around }\end{array}$ & n.a. & 6 & 57.83 & 24.67 & No selection & \\
\hline 10 & $\begin{array}{l}\text { Right temporal } \\
\text { lobe }\end{array}$ & right $\mathrm{HS}$ & $\begin{array}{l}\text { bitemporal } \\
(\mathrm{L}>\mathrm{R})\end{array}$ & left temporal & $\mathrm{HS}$ & 6 & 64.00 & 18.00 & Not informative & Right \\
\hline 11 & $\begin{array}{l}\text { Right temporal } \\
\text { lobe }\end{array}$ & Right HS & right temporal & Right temporal & HS & 4 & 57.75 & 21.75 & Correct & Right \\
\hline 12 & $\begin{array}{l}\text { Right temporal } \\
\text { lobe }\end{array}$ & Normal & $\begin{array}{l}\text { right temporal } \\
\text { and insula }\end{array}$ & n.a. & $\begin{array}{l}\text { End-folium scle- } \\
\text { rosis }\end{array}$ & 4 & 52.40 & 16.00 & Correct & Right \\
\hline 13 & Left frontal lobe & Normal & $\begin{array}{l}\text { left insula, left } \\
\text { frontal and mid- } \\
\text { line frontal }\end{array}$ & n.a. & n.a. & 3 & 56.33 & 24.00 & Correct & Left \\
\hline 14 & $\begin{array}{l}\text { Left amygdala \& } \\
\text { hyppocampus }\end{array}$ & $\begin{array}{l}\text { left temporal sur- } \\
\text { gical lesion and } \\
\text { gliosis, HS }\end{array}$ & n.a & left temoporal & HS, gliosis & 6 & 57.67 & 22.00 & Not informative & Left \\
\hline 15 & $\begin{array}{l}\text { Right temporal } \\
\text { lobe }\end{array}$ & $\begin{array}{l}\text { anterior temporal } \\
\text { FCD }\end{array}$ & n.a. & n.a. & FCD & 2 & 65.00 & 09.00 & No selection & \\
\hline 16 & Left frontal lobe & Normal & inconclusive & n.a. & n.a. & 2 & 56.00 & 17.5 & No selection & \\
\hline 17 & Right frontal lobe & right frontal FCD & inconclusive & $\begin{array}{lr}\text { right } & \text { frontal } \\
\text { and } & \text { bilateral } \\
\text { ventrotemporal }\end{array}$ & $\begin{array}{l}\text { gianeuronal } \\
\text { tumour }\end{array}$ & 6 & 55.83 & 12.67 & Not informative & Left \\
\hline 18 & $\begin{array}{l}\text { Left temporal } \\
\text { lobe }\end{array}$ & $\begin{array}{l}\text { cystic structure } \\
\text { left hippocampus }\end{array}$ & n.a. & left temporal & ganglioglioma & 6 & 52.50 & 16.00 & No selection & \\
\hline
\end{tabular}

\footnotetext{
${ }^{a}$ outside the brain

$b$ selected ICs contradict

${ }^{c}$ multiple clusters in IC
} 


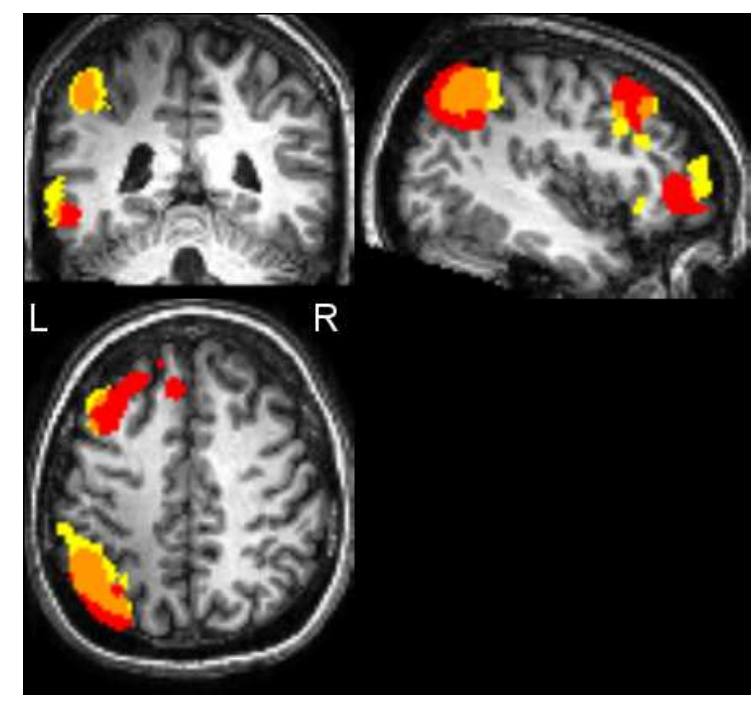

(a)

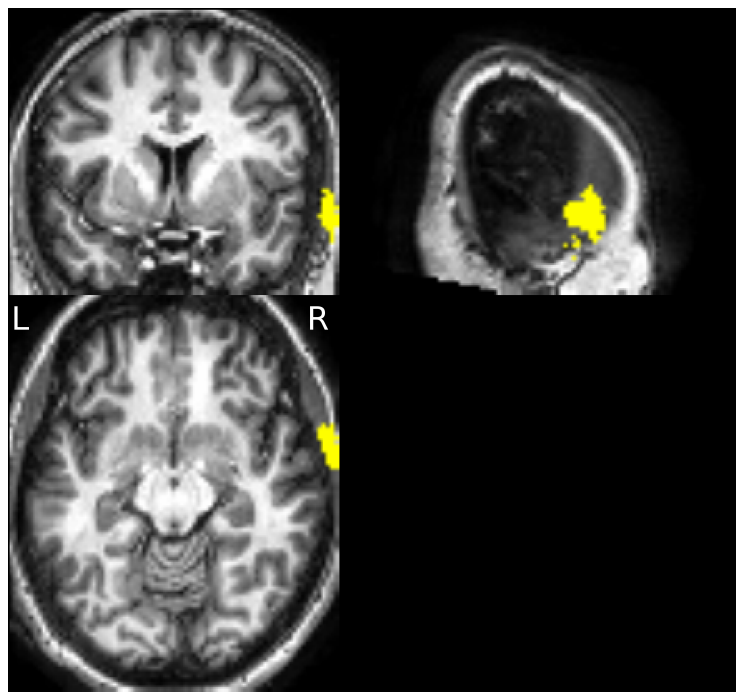

(c)

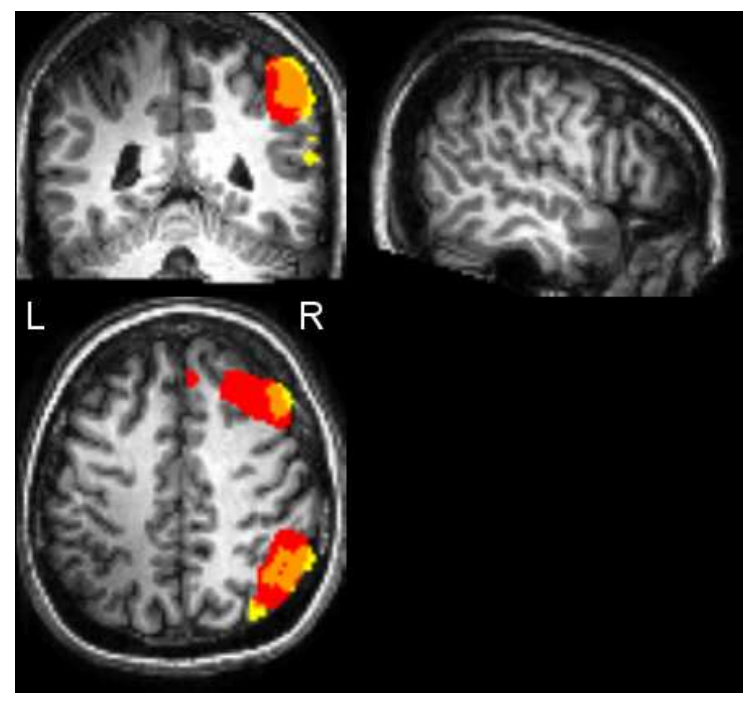

(b)

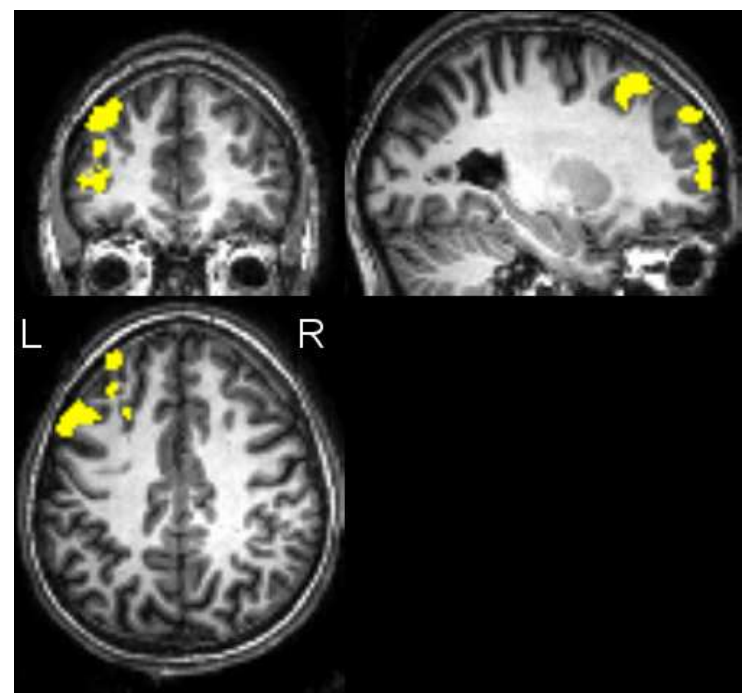

(d)

Figure 3: Non-informative IC maps selected in patients and a selected IC in a control subject. (a) (b) In 6 patients the selected IC maps showed suprathreshold clusters in areas related to the executive control network (ECN). A group average map using left and right lateralised maps and the left and right ECN are shown in yellow and red, respectively. The overlap between these maps is indicated in orange. (c) In one patient the selected IC showed a cluster outside the brain. The map is not informative. (d) Selected IC in a control subject. A selection was made in 1 out of 13 cases. 
tional EEG-fMRI analysis. Instead, we aim to offer an alternative in those cases where the latter fails due to the lack of visually detectable spikes. In this sense our approach has a high added value as it extends the applicability of fMRI to the EEG-negative patient group. This is a crucial difference between our study and the one described in (Zhang et al. (2014)), where this aspect cannot be judged due to the lack of simultaneously recorded EEG. For the same reason, (Zhang et al. (2014)) needs to deliver localization information in all fMRI studies, including the ones which might be EEGpositive in case EEG was recorded. Therefore, the sensitivity of (Zhang et al. (2014)) and our method should not be compared directly.

Even though recording EEG inside the magnetic field of the MR scanner poses technical challenges, there is a considerable amount of EEG-positive cases, where EEG-correlated fMRI analysis remains a viable option. Still, even if good quality EEG is available, marking of interictal events is a time consuming, subjective, raterdependent process (Zijlmans et al. (2007)). As the erroneous marking of interictal events affects the outcome of EEG-fMRI analysis (Flanagan et al. (2009)), an objective (semi-)automation of the spike identification could have high benefits. A novel spike templatebased semi-automatic method was shown to outperform existing techniques (Grouiller et al. (2011)) in EEGpositive cases. It would be worthwhile to investigate how its performance compares to our approach in EEGnegative cases, and perhaps a combination of both techniques could yield higher sensitivity in this crucial patient group.

The approach presented in this study has certain limitations. From the clinical point of view, the proposed approach is not helpful in case of a diffuse epileptogenic zone or multifocal epilepsies. Instead, it is only applicable in cases with a single, lateralised focus. The presence of a single focus can be established using other modalities. Semiology of seizures, MRI, ictal EEG and FDG-PET may suggest a single focus, but spikes on interictal EEG may not be present. We believe that our method may be useful in this clinical setting, and may provide added value.

Further, in order to be able to objectively detect the localization of the EZ, we only consider maps containing a single activation cluster as informative. On one hand, epilepsy being a network disorder, it is plausible to find multiple areas of BOLD activation related to epileptic activity. Thus, the above choice may seem unfounded. On the other hand, based on our experience, even in cases where EEG-fMRI shows multiple areas of BOLD activation, these activation clus- ters are represented in different independent components, representing different aspects of epileptic activity [21]. Similar findings were reported in a recent study (van Houdt et al. (2014)). The authors argued that in patients where the EEG-fMRI patterns included more than one activation cluster, one of them reflected the onset area, while others were related to propagation. Furthermore, they confirmed that ICA separated these different areas in different components. Finally, the authors reported an example where such additional components seemed to overlap with standard resting state networks. These findings suggest that ICA and GLM-based EEGfMRI capture different hemodynamic phenomena. Indeed, spatial independent component analysis models the data as a linear mixture of processes which are statistically independent in space. However, GLM-based analysis looks for voxels which covary with the timing of interictal epileptic discharges. The mismatch between investigating spatial versus temporal phenomena can contribute to the difference in the analysis outcome, which renders these approaches partly complementary.

There are limitations from the methodological point of view as well. Our approach is based on a supervised learning technique, i.e. the goodness of our model largely depends on the quality of the training dataset. We see two factors limiting the current performance, which could be improved in future work. First, the classifier is trained on data from EEG-positive cases, and applied on EEG-negative ones. As such, we are looking for BOLD signal characteristics related to epileptic neural activity, which are consistent regardless of whether this neural activity leads to electrographical changes. However, it is likely that different types of interictal activity are present in EEG-positive and EEGnegative cases, and these will cause different BOLD signal changes. Higher performance can be expected in case the training and testing dataset better resemble each other. Therefore, future work will aim at training a classifier on EEG-negative cases and testing it on a new, independent validation set. Moreover, a larger training set is expected to yield improved results as well. Compared to our previous study (Hunyadi et al. (2014)), where only 9 patients were included in the training set instead of 12 , the specificity of our method has increased from $77 \%$ to $92 \%$. Note, however, that this improvement could partially be attributed to the fact that we were more rigorous about the inclusion criteria. As opposed to (Hunyadi et al. (2014)), all the runs included in the current study had the same length, rendering the withinclass variability of some sensitive features lower.

For the interpretation of the selected IC maps, we propose to use a z-threshold of 5 and the minimal cluster 
size of 100 voxels. These values were chosen somewhat arbitrarily. Therefore, we have investigated the effect of slightly varying these parameters. At a z-threshold of 4 the selected IC of patient 12 contained 2 additional clusters at the brainstem and in the contralateral temporal lobe. On the other hand, at a z-threshold of 6 the activation cluster of the selected IC in patient 1 and 11 was split into 2 clusters. However, as these clusters still point to the same anatomical region, this does not influence the interpretation. Further, the selected non-informative map in patient 14 shows only 1 activation cluster at a threshold of 6 , rendering it an informative but incorrect map. The minimal cluster size can be varied between 90 and 140 without any influence on the results. A minimal cluster size chosen below and above these values has similar effects as varying the $\mathrm{z}$ threshold. In summary, the final outcome is sufficiently robust against perturbations in the chosen parameters.

In 6 cases IC maps resembling the executive control network (ECN) were selected. It is probably selected by our algorithm due to the fact that this network tends to lateralise. The presented method could be improved by ruling out such maps based on their similarity to predefined reference atlases. However, in case the epileptogenic zone is located at a region which is part of such a reference atlas, the epileptic ICs might also be removed this way. Previous studies have reported the impairment of the ECN in mesial temporal lobe epilepsy (Zhang et al. (2009)), as well as an abnormal causal connectivity, i.e. an increased driving effect of the ECN to the epileptogenic zone, compared to controls (Ji et al. (2013)). Notably, in our dataset a majority of these maps were highly lateralised ipsilateral to the EZ. Therefore, although these maps are not directly informative to the localisation, they could potentially indicate the laterality of the EZ. However, before applying such considerations in clinical practice for formulating surgical indications, these findings should be confirmed using a larger patient group.

We have demonstrated that our approach can find epileptic IC maps prospectively in EEG-negative cases. This suggests the presence of interictal activity during the time of the recording despite the fact that this activity is invisible in the EEG. Indeed, the hippocampus often shows spiking which is unnoticeable on scalp EEG. Mesial temporal lobe epilepsies, nevertheless, are easy to diagnose with conventional techniques such as ictal EEG, MRI and FDG-PET. However, we argue that our method can detect epileptic maps in different patient groups as well. One could hypothesise that patients with less straightforward mesial TLE (e.g. a patient with multiple lesions or with normal MRI) will also demonstrate hidden epileptic activity in the pathologic hippocampus. This was actually the case with $\mathrm{Pa}-$ tient 12 who had a normal MRI and end-folium sclerosis was indicated during the pathological examination. Moreover, the other two cases where the proposed method made a correct selection (Patient 1 with neocortical TLE and Patient 13 with neocortical ETLE) demonstrate that this hidden epileptic activity can also be found in structures outside the hippocampus. We would like to stress that Patient 12 and 13 had a normal MRI. Therefore, the EZ in these two cases could not be determined using conventional clinical tools and the decision was facilitated by SISCOM. Patient 13 with MR-negative frontal lobe epilepsy represented a particularly interesting case. In general, the success rate of frontal lobe epilepsy surgery is much lower than following temporal lobectomy (Jeha et al. (2007)). Within this already challenging group, MR-negative cases are at higher risk of recurrence after surgery (83\%) compared to lesional cases (45\%) (Jeha et al. (2007)). In patient 13 , guided by SISCOM findings, intracranial grids were implanted which helped to delineate the EZ, and lead to a successful surgery (ILAE class 1 outcome with 3 years follow-up). Notably, the epileptic IC map selected by our method indicated a single activation cluster, which coincides with the actual resection zone in the left frontal lobe. Hence, our method could correctly identify the EZ non-invasively, solely based on fMRI and was better than the other non-invasive methods of investigation, such as interictal and ictal EEG and SISCOM. In summary, these cases demonstrate that the proposed method can be informative and have an added value in clinical decision making.

\section{Conclusion}

We presented a novel approach to infer to the localisation of interictal epileptic sources based on the fMRI recordings alone. As such, it can extend the applicability of fMRI recordings to patients where traditional EEG-fMRI analysis cannot be carried out. Moreover, we have reported cases where our fMRI analysis has added value in the clinical decision making compared to conventional techniques. The significance of the technique lies within the fact that it is very specific and fully automated, therefore, it can be used prospectively in clinical practice.

\section{Acknowledgement}

Research Council KUL: CoE PFV/10/002 (OPTEC); PhD/Postdoc grants; Flemish Government: FWO: 
projects: G.0427.10N (Integrated EEG-fMRI), G.0108.11 (Compressed Sensing) G.0869.12N (Tumor imaging) G.0A5513N (Deep brain stimulation); $\mathrm{PhD} /$ Postdoc grants; IWT: projects: TBM 080658-MRI (EEG-fMRI), TBM 110697-NeoGuard; PhD/Postdoc grants; iMinds Medical Information Technologies SBO 2015, ICON: NXT_Sleep; Flanders Care: Demonstratieproject Tele-Rehab III (2012-2014); Belgian Federal Science Policy Office: IUAP P7/19/ (DYSCO, "'Dynamical systems, control and optimization",, 2012-2017); Belgian Foreign Affairs-Development Cooperation: VLIR UOS programs; EU: The research leading to these results has received funding from the European Research Council under the European Union's Seventh Framework Programme (FP7/20072013) / ERC Advanced Grant: BIOTENSORS ( ${ }^{\circ}$ 339804).This paper reflects only the authors' views and the Union is not liable for any use that may be made of the contained information. Other EU funding: RECAP 209G within INTERREG IVB NWE programme, EU MC ITN TRANSACT 2012 ( $\mathrm{n}^{\mathrm{O}} 316679$ ), ERASMUS EQR: Community service engineer $\left(\mathrm{n}^{\mathrm{o}}\right.$ 539642-LLP-1-2013)

\section{Appendix}

The following features were extracted from the fMRI ICs for the purpose of the second discrimination step, aiming at selecting epileptic ICs from all BOLD signal related ICs. The motivation behind these features is explained in section 2.3 Below the mathematical definition of the features are given.

\subsection{Number of clusters}

The z-scored spatial IC maps were thresholded at a value of 5 . Then, neighboring suprathreshold voxels were grouped together to form clusters. The value of this feature is set to the number of clusters comprising at least 100 voxels.

\subsection{Asymmetry}

The asymmetry of an IC spatial map is assessed by the following formula:

$$
A A=\left|\sum_{i=1}^{H}\left(v_{i}^{(l)}-v_{i}^{(r)}\right)\right|,
$$

where $v_{i}^{(l)}$ denotes the z-scored value of the $i^{t h}$ voxel in the left hemisphere, $v_{i}^{(r)}$ denotes the z-scored value of the corresponding contralateral voxel and $H$ is the total number of voxels in one hemisphere.

\subsection{Sparsity in activelet basis}

The neural activity of interest consists of $k$ interictal epileptic spikes with amplitudes $A_{k}$ and onsets $t_{k}$. The fMRI measures the BOLD signal changes as a result of this neural activity. The hemodynamic system linking the neural activity to the BOLD signal is denoted by $h$, and is commonly assumed to be linear and shiftinvariant. The BOLD signal recorded by the fMRI can be written as follows:

$$
y(t)=\sum_{k} A_{k} h\left(t-t_{k}\right)+\varepsilon(t),
$$

where $\varepsilon(t)$ is an unknown noise term comprising noise, baseline, drifts, physiological artefacts and possibly other unrelated neural activity. The goal is to recover the activity of interest from the noisy signal. Given that the neural events are sparse in time, the linearity assumption holds and the transient BOLD signals can be sparsely represented in the so-called activelet basis.

Activelets are a dictionary of wavelet basis functions constructed based on the linear approximation of the balloon model of the hemodynamic response function. More specifically, the (approximated) hemodynamic system linking the neural stimulus and the BOLD signal can be expressed with a differential operator. The mathematical construction of wavelets corresponding to such linear time-invariant differential equations is described in (Khalidov et al. (2011)) and (Khalidov and Unser (2006)). 3

Let $\phi$ be the overcomplete dictionary matrix containing the basis functions of the undecimated activelet transform. Then the estimated neural activity $y$ will be given by $y=\phi \beta_{0}$, where $\beta_{0}$ is the solution of the convex $l_{1}$ optimization problem:

$$
\min _{\beta}\left(\frac{1}{2}\|y-\phi \beta\|_{2}^{2}+\lambda\|\beta\|_{1}\right)
$$

The regularization parameter $\lambda$ controls the trade-off between the sparsity of the solution and the reconstruction error, a higher value favouring a sparser solution. The value of $\lambda$ was set to 2.5 based on preliminary experiments on the training data. $\phi$ is the overcomplete dictionary matrix containing the basis functions of the undecimated activelet transform. It is a matrix of size $T \times P$, where $\mathrm{T}$ is the length of the time series and $P=3 \cdot T$ as the number of wavelet decomposition scales was set to 3 (Lopes et al. (2012)). The minimization

\footnotetext{
${ }^{3}$ The Matlab code implementing the activelet dictionary was kindly provided by Dimitri Van de Ville, MIPLab, EPFL and University of Geneva
} 
problem in (5) was solved by the Homotopy algorithm (Donoho and Tsaig (2008)).

The time course of an epilepsy related IC is expected to have a sparser representation in the activelet basis compared to non-related ICs. The sparsity of the representation in the activelet basis was quantified with the Gini index (Hurley and Rickard (2008)), which measures the statistical dispersion of the magnitude of the coefficients.

\subsection{Sparsity in sine basis}

A sine dictionary $\Phi_{k}(t)$ is constructed as:

$$
\Phi_{k}(t)=\sin (2 \pi k t) k=1,2, \ldots, \frac{N}{2} 0 \leq t \leq 1
$$

This dictionary was restricted to $\left\{\Phi_{k}(t), k=6 \ldots 68\right\}$ in order to match the expected $0.01-0.1 \mathrm{~Hz}$ frequency of BOLD signals, sampled at $\mathrm{N}=270$ points every $2.5 \mathrm{sec}-$ onds. The basic matching pursuit algorithm, available in Matlab, was used to retrieve the coefficients corresponding to the best nonlinear approximation of the fMRI IC. Again, the sparsity of the representation was quantified with the Gini index.

\section{References}

An, D., Fahoum, F., Hall, J., Olivier, A., Gotman, J., Dubeau, F., 2013. Electroencephalography/functional magnetic resonance imaging responses help predict surgical outcome in focal epilepsy. Epilepsia 54, 2184-2194.

Blume, W.T., Lüders, H.O., Mizrahi, E., Tassinari, C., van Emde Boas, W., Engel, J., 2001. Glossary of descriptive terminology for ictal semiology: Report of the ILAE task force on classification and terminology. Epilepsia 42(9), 1212-8.

Buxton, R., Wong, E., Frank, L., 1998. Dynamics of blood flow and oxygenation changes during brain activation: The balloon model. Magnetic Resonance in Medicine 39, 855-864.

Cordes, D., Haughton, V., Arfanakis, K., Carew, J., Turski, P., Moritz, C., Quigley, M., Meyerand, M., 2001. Frequencies contributing to functional connectivity in the cerebral cortex in resting-state data. American Journal of Neuroradiology 22, 1326-1333.

De Boer, H.M., Mula, M., Sander, J.W., 2008. The global burden and stigma of epilepsy. Epilepsy Behav 12, 540 - 6.

De Martino, F., Gentile, F., Esposito, F., Balsi, M., Di Salle, F., Goebel, R., Formisano, E., 2007. Classification of fMRI independent components using IC-fingerprints and support vector machine classifiers. NeuroImage 34, 177 - 194.

Donoho, D., Tsaig, Y., 2008. Fast solution of $\ell_{1}$-norm minimization problems when the solution may be sparse. Information Theory, IEEE Transactions on 54, 4789-4812.

Engel, J., 1996. Surgery for seizures. N Engl J Med 334, 647-53.

Fisher, R.S., van Emde Boas, W., Blume, W., Elger, C., Genton, P., Lee, P., Engel, J., 2005. Epileptic seizures and epilepsy: Definitions proposed by the international league against epilepsy (ILAE) and the international bureau for epilepsy (IBE). Epilepsia 46, 470472.
Flanagan, D., Abbott, D.F., Jackson, G.D., 2009. How wrong can we be? the effect of inaccurate mark-up of EEG/fMRI studies in epilepsy. Clinical Neurophysiology 120, 1637-1647.

Foldvary, N., Klem, G., Hammel, J., Bingaman, W., Najm, I., Lüders, H., 2001. The localizing value of ictal EEG in focal epilepsy. Neurology 57, 2022-2028.

Gotman, J., Kobayashi, E., Bagshaw, A.P., Bénar, C.G., Dubeau, F., 2006. Combining EEG and fMRI: A multimodal tool for epilepsy research. Journal of Magnetic Resonance Imaging 23, 906-920.

Grouiller, F., Thornton, R.C., Groening, K., Spinelli, L., Duncan, J.S., Schaller, K., Siniatchkin, M., Lemieux, L., Seeck, M., Michel, C.M., Vulliemoz, S., 2011. With or without spikes: localization of focal epileptic activity by simultaneous electroencephalography and functional magnetic resonance imaging. Brain 134, 28672886.

Hamandi, K., Salek Haddadi, A., Liston, A., Laufs, H., Fish, D., Lemieux, L., 2005. fMRI temporal clustering analysis in patients with frequent interictal epileptiform discharges: comparison with EEG-driven analysis. Neuroimage 26, 309-316.

Hunyadi, B., Tousseyn, S., Dupont, P., Van Huffel, S., Van Paesschen, W., De Vos, M., 2014. Automatic selection of epileptic independent fMRI components, in: Engineering in Medicine and Biology Society (EMBC), 2014 36th Annual International Conference of the IEEE, pp. 1-4.

Hunyadi, B., Tousseyn, S., Mijović, B., Dupont, P., Van Huffel, S., Van Paesschen, W., De Vos, M., 2013. Ica extracts epileptic sources from fMRI in EEG-negative patients: A retrospective validation study. PLoS ONE 8, e78796.

Hurley, N., Rickard, S., 2008. Comparing measures of sparsity, in: Machine Learning for Signal Processing, 2008. MLSP 2008. IEEE Workshop on, pp. 55 -60.

Jeha, L.E., Najm, I., Bingaman, W., Dinner, D., Widdess-Walsh, P., Lüders, H., 2007. Surgical outcome and prognostic factors of frontal lobe epilepsy surgery. Brain 130, 574-584.

Ji, G.J., Zhang, Z., Zhang, H., Wang, J., Liu, D.Q., Zang, Y.F., Liao, W., Lu, G., 2013. Disrupted causal connectivity in mesial temporal lobe epilepsy. PloS one 8, e63183.

Khalidov, I., Fadili, J., Lazeyras, F., Van De Ville, D., Unser, M., 2011. Activelets: Wavelets for sparse representation of hemodynamic responses. Signal Processing 91, 2810 - 2821.

Khalidov, I., Unser, M., 2006. From differential equations to the construction of new wavelet-like bases. Signal Processing 54, 12561267.

Leite, M., Leal, A., Figueiredo, P., 2013. Transfer function between EEG and BOLD signals of epileptic activity. Frontiers in Neurology $4,-$.

LeVan, P., Tyvaert, L., Moeller, F., Gotman, J., 2010. Independent component analysis reveals dynamic ictal BOLD responses in EEG-fMRI data from focal epilepsy patients. NeuroImage 49, $366-378$.

Lopes, R., Lina, J., Fahoum, F., Gotman, J., 2012. Detection of epileptic activity in fMRI without recording the EEG. NeuroImage 60, $1867-1879$.

Moeller, F., LeVan, P., Gotman, J., 2011. Independent component analysis (ICA) of generalized spike wave discharges in fMRI: Comparison with general linear model-based EEG-fMRI. Human Brain Mapping 32, 209-217.

Morgan, V., Li, Y., Abou-Khalil, B., Gore, J., 2008. Development of 2dTCA for the detection of irregular, transient BOLD activity. Human Brain Mapping 29, 57-69.

Morgan, V., Price, R., Arain, A., Modur, P., Abou-Khalil, B., 2004. Resting functional MRI with temporal clustering analysis for localization of epileptic activity without EEG. NeuroImage 21, 473 $-481$.

Nonclercq, A., Foulon, M., Verheulpen, D., De Cock, C., Buzatu, M., 
Mathys, P., Van Bogaert, P., 2012. Cluster-based spike detection algorithm adapts to interpatient and intrapatient variation in spike morphology. Journal of neuroscience methods 210, 259-265.

Panayiotopoulos, C.P., 2005. The Epilepsies: Seizures, Syndromes and Management. Oxfordshire (UK): Bladon Medical Publishing.

Rodionov, R., De Martino, F., Laufs, H., Carmichael, D., Formisano, E., Walker, M., Duncan, J., Lemieux, L., 2007. Independent component analysis of interictal fMRI in focal epilepsy: Comparison with general linear model-based EEG-correlated fMRI. NeuroImage $38,488-500$.

Rosenow, F., Lüders, H., 2001. Presurgical evaluation of epilepsy. Brain 124, 1683-1700.

Salek-Haddadi, A., Diehl, B., Hamandi, K., Merschhemke, M., Liston, A., Friston, K., Duncan, J., Fish, D., Lemieux, L., 2006. Hemodynamic correlates of epileptiform discharges: An EEG-fMRI study of 63 patients with focal epilepsy. Brain Research $1088,148-166$.

Salimi-Khorshidi, G., Douaud, G., Beckmann, C.F., Glasser, M.F., Griffanti, L., Smith, S.M., 2014. Automatic denoising of functional MRI data: Combining independent component analysis and hierarchical fusion of classifiers. NeuroImage 90, 449-68.

Shirer, W., Ryali, S., Rykhlevskaia, E., Menon, V., Greicius, M., 2012. Decoding subject-driven cognitive states with whole-brain connectivity patterns. Cerebral cortex 22, 158-165.

Thornton, R., Rodionov, R., Laufs, H., Vulliemoz, S., Vaudano, A. Carmichael, D., Cannadathu, S., Guye, M., McEvoy, A., Lhatoo, S., Bartolomei, F., Chauvel, P., Diehl, B., De Martino, F., Elwes, R., Walker, M., Duncan, J., Lemieux, L., 2010. Imaging haemodynamic changes related to seizures: Comparison of EEG-based general linear model, independent component analysis of fMRI and intracranial EEG. NeuroImage 53, 196 - 205.

Thornton, R., Vulliemoz, S., Rodionov, R., Carmichael, D., Chaudhary, U., Diehl, B., Laufs, H., Vollmar, C., McEvoy, A., Walker, M., Bartolomei, F., Guye, M., Chauvel, P., Duncan, J., Lemieux, L., 2011. Epileptic networks in focal cortical dysplasia revealed using electroencephalographyunctional magnetic resonance imaging. Annals of Neurology 70, 822-837.

Tousseyn, S., Dupont, P., Goffin, K., Sunaert, S., Van Paesschen, W., 2014a. Sensitivity and specificity of interictal EEG-fMRI for detecting the ictal onset zone at different statistical thresholds. Frontiers in Neurology 5, 279-311.

Tousseyn, S., Dupont, P., Robben, D., Goffin, K., Sunaert, S., Van Paesschen, W., 2014b. A reliable and time-saving semi-automatic spike template-based analysis of interictal EEG-fMRI. Epilepsia, in press.

van Houdt, P.J., Ossenblok, P., Colon, A., Hermans, K., Verdaasdonk, R., Boon, P., de Munck, J., 2014. Are epilepsy-related fmri components dependent on the presence of interictal epileptic discharges in scalp EEG? Brain topography , 1-13.

Zhang, C.H., Lu, Y., Brinkmann, B., Welker, K., Worrell, G., He, B., 2014. Lateralization and localization of epilepsy related hemodynamic foci using presurgical fMRI. Clinical Neurophysiology, in press.

Zhang, Z., Lu, G., Zhong, Y., Tan, Q., Yang, Z., Liao, W., Chen, Z., Shi, J., Liu, Y., 2009. Impaired attention network in temporal lobe epilepsy: a resting fMRI study. Neuroscience letters 458, 97-101.

Zijlmans, M., Huiskamp, G., Hersevoort, M., Seppenwoolde, J.H., van Huffelen, A.C., Leijten, F.S.S., 2007. EEG-fMRI in the preoperative work-up for epilepsy surgery. Brain 130, 2343-2353. 